\title{
Toy Story: what I have learned from playing with toys about the physics of living cells
}

Robert Austin

Robert H. Austin, "Toy Story: what I have learned from playing with toys about the physics of living cells," Proc. SPIE 7929, Microfluidics, BioMEMS, and Medical Microsystems IX, 792902 (11 February 2011); doi: 10.1117/12.882723 


\title{
Toy Story: What I have learned from playing with toys about the physics of living cells
}

\author{
Robert H. Austin ${ }^{a}$ \\ ${ }^{a}$ Princeton University, Dept. of Physics, Princeton, NJ USA
}

\begin{abstract}
Yogi Berra once noted that "You can observe a lot just by watching." A similar remark can be made about toys: you can learn a lot of physics by playing with certain children's toys, and given that physics also applies to life, you could hope that it would also be possible to learn about the physics of living cells by close observation of toys, loosely defined. I'll start out with a couple of toys, rubber duckies and something called a soliton machine and discuss insights (or failures) in how "energy" moves in biological molecules. I'll bring back the rubber duckies and a toy suggested by one of the eccentrics known to roam the halls of academia to discuss how this lead to studies how cells move and collective aspects of cell movement. Then I'll talk about mazes and how they lead to experiments on evolution and cancer. Hopefully this broad range of toys will show how indeed "You can observe a lot just by watching" about some of the fundamental physics of living cells.
\end{abstract}

Keywords: microfabrication, nanofabrication, ecology, evolution,

\section{INTRODUCTION}

The scale of physics aspects of living cells covers an enormous length scale, from nanometers (nm) to meters. We will in this paper almost cover that range, starting with one of the very basic problems in biological physics (how energy moves in a biomolecule coherently), will move on to how bacteria swim, talk about the social lives of bacteria and how it connects to cancer, and mention at the end the latest toy craze which I claim was stolen from me. The connection with toys comes from the very nature of toys: they typically surprise and delight even adults because often the behave in surprising and unexpected ways by clever uses of physics principles. I view living systems in the same way: they act in often completely astonishing and unexpected ways, but they must work within the constraints of the physics we teach our freshmen students.

The first part of the talk is how then toys can be deceptive. The toy here is actually one we made and I am afraid will never be a best seller because it is rather boring, but it is connected to a well known toy called the chaotic pendulum. The chaotic pendulum in its simplest form is just a double pendulum: the end of one pendulum acts as the pivot point for a second pendulum (see Fig. 1, taken from http://en.wikipedia.org/wiki/File: DPLE. jpg. The surprising thing about the chaotic pendulum is that if the the upper pendulum is released from a range of certain large angles (so the motion is decidedly anharmonic) the motion quickly becomes indeterminate: the motion shows a sensitive dependence on initial conditions and seemingly randomly exchanges energy with no clear secondary frequencies. The connection with biomolecules is this: molecular motors are the protein marvels that take chemical free energy from ATP and turn that into functional motion, such as the kinesin motor that transports cargo by moving along microtubules. Normally, in chemistry when a reaction occurs such as the exothermic hydrolysis of ATP which releases about $50 \mathrm{~kJ} \mathrm{~mol}^{? 1}$ (about $0.5 \mathrm{eV}$ ), the net result is that the surrounding atoms get warm, that is the free energy mostly goes into increasing the entropy of the surrounds because of all the anharmonic coupling that makes the motion fundamentally chaotic and irreversible in time. The chaotic pendulum is a good toy because it shows at a macroscopic scale this fundamental irreversibility of an harmonic motion.

But molecular motors don't just get hot when they hydrolyze ATP, they actually do directed physical work! How is that possible? We don't really understand this, but the toy we made can help demonstrate this. This toy is also coupled anharmonic pendula, but instead of being connected serially the pendula are connected in

Further author information: (Send correspondence to R.H.A.)

R.H.A..: E-mail: austin@princeton.edu, Telephone: 16092584353

Microfluidics, BioMEMS, and Medical Microsystems IX, edited by Holger Becker, Bonnie L. Gray, Proc. of SPIE Vol. 7929, $792902 \cdot$ (c) 2011 SPIE · CCC code: 0277-786X/11/\$18 · doi: 10.1117/12.882723

Proc. of SPIE Vol. $7929792902-1$ 


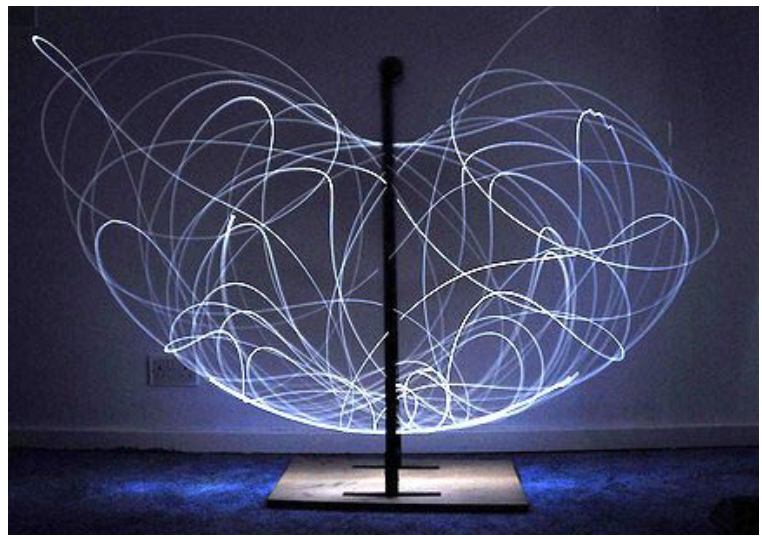

Figure 1. Double Pendulum long exposure, tracked with LED light at its end.

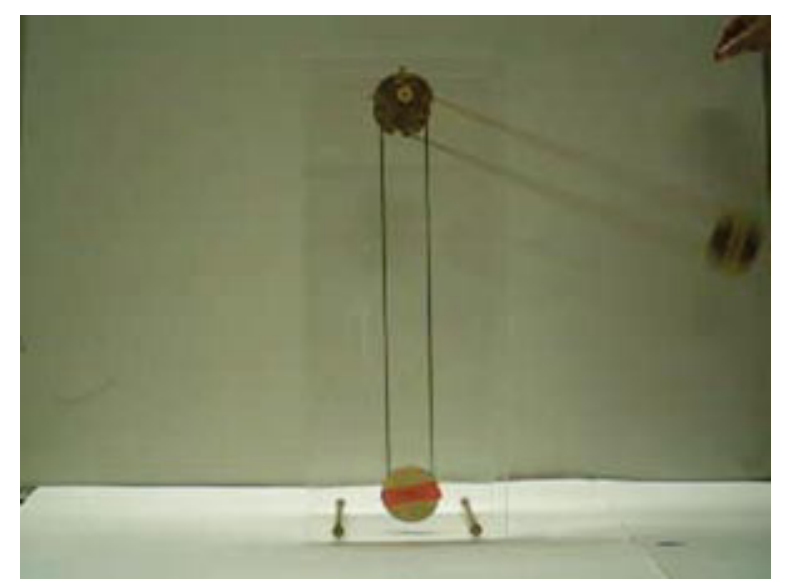

Figure 2. Snap-shot of the motion of 2 pendula coupled by a linear torsional spring. Energy stays trapped in the moving pendula.

parallel by a weak linear torsional spring, so that the two pendula slow exchange energy in a perturbative manner through the torsional coupling. In a sense this is more realistic then the serial coupled pedulua since that system has no real isolated mode, while in reality protein secondary structures such as the $\alpha$ helix show a well defined mode with $\mathrm{Q}$ factor of about 50, better even then the mechanical device shown in Fig. 1. The interesting thing about this toy is that in spite of the coupling between two pendula virtually all the energy stays trapped in one of the pendulua IF that pendulum is moving with a maximum angle $\theta_{o}$ large enough that it is effectively "detuned" out of the bandwidth of the un-excited pendulum. In that case the constantly changing phase shift of the large angle pendulum simply takes energy into and out of the other system with no net transfer.

One could hope then that this toy demonstrates the ability of the free energy of hydrolysis to be trapped in the molecule motor and be later used for doing mechanical work. But it is probably wrong, because it is a mistake to think that the free energy of hydrolysis, which is a true thermodynamic free energy with many degrees of freedom, can be turned into a single degree of freedom, namely the movement of a single quantum oscillator. To do that would imply that the entropy of the system had vastly decreased spontaneously, and that violates the second law of thermodynamics. We have discussed in a previous paper ${ }^{1}$ the fundamental problem that existed in conflation of single degrees of freedom with the thermodynamics of many degrees of freedom and how theory can be lead astray be assuming that free energy contained in many degrees of freedom can be transferred to a single degree of freedom. The toy in this case fools you.

\section{RATCHETS}

When I first came to Princeton as an Assistant Professor my mentor John Hopfield came down after a few days and told me about a visitor that came by with an idea to make money. The visitor's idea was that he wanted to make a membrane with funnel-shaped holes in it. idea was that the funnels would channel molecules from the open end to the closed end under the influence of brownian motion, concentrating the molecules on the narrow side of the membrane without any work being done by an outside force. Thus, at now cost biomolecules could be concentrated! Obviously, this would violate the 2nd Law of thermodynamics once again, since the entropic density of a gas scales as the log of the density of the molecules, the net effect of such a membrane would be to decrease the entropy of the universe. I was intrigued by the idea however and built a simple mechanical version of the visitor's idea. For molecules I used little aluminum rods, and the funnels were big enough at the wide end for the rods to come in in any orientation, but they had to be aligned to pass through the narrow end. Fig. 3 shows what it looked like. For thermal motion I just tried to randomly shake the device. In fact, as you might expect from fly traps and lobster traps, with shaping the device concentrated the "molecules". A violation of the Second Law: unlikely since my shaking is hardly thermal white noise in terms of the power spectrum.

However, a post-doc in my lab at the time, Peter Galajda, decided to scale the device down to a more interesting scale, namely to the micron scale and instead of shaken metal rods try for randomly swimming 


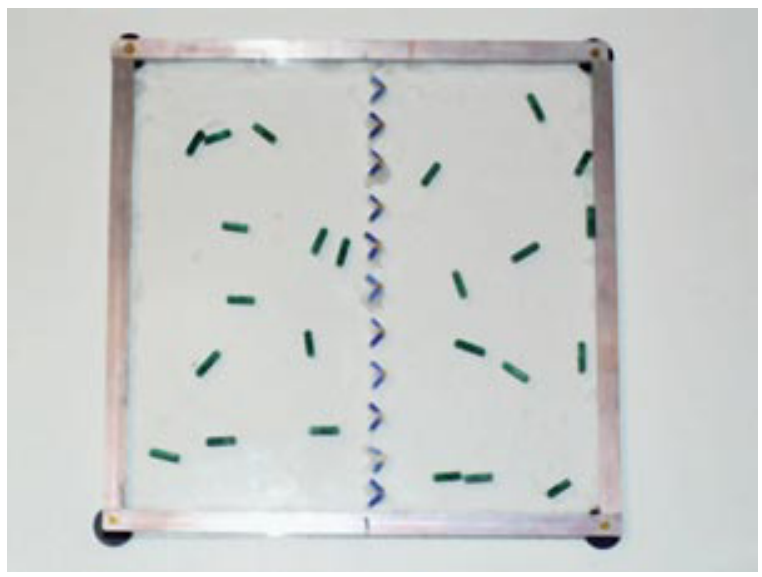

Figure 3. The constructed funnel line before shaking. The aluminum "molecules" are painted green.

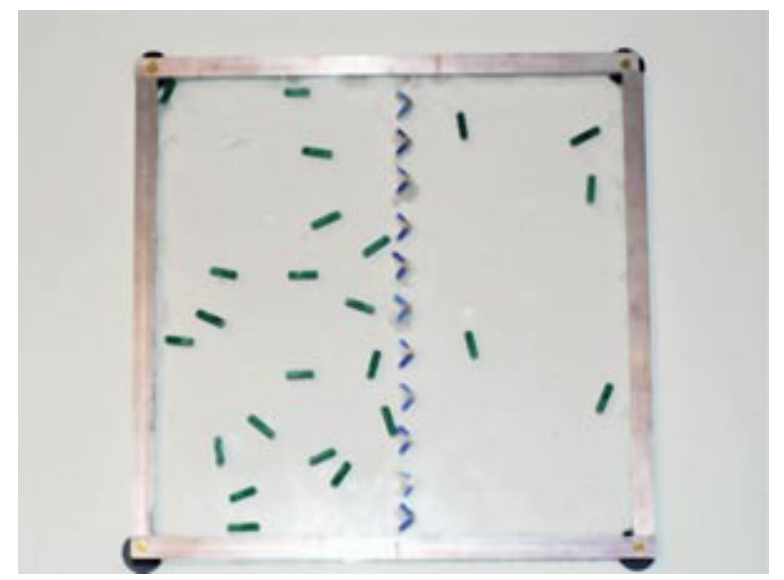

Figure 4. After shaking. The "molecules" were indeed concentrated.

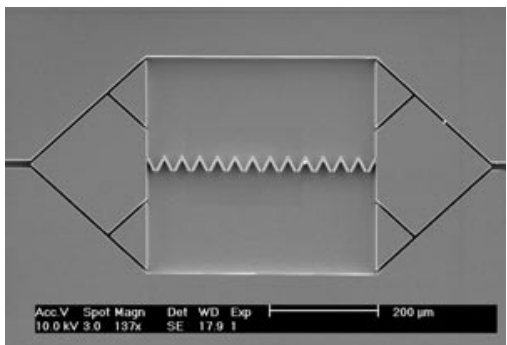

Figure 5. SEM image of a microfabricated array of funnels for bacteria.

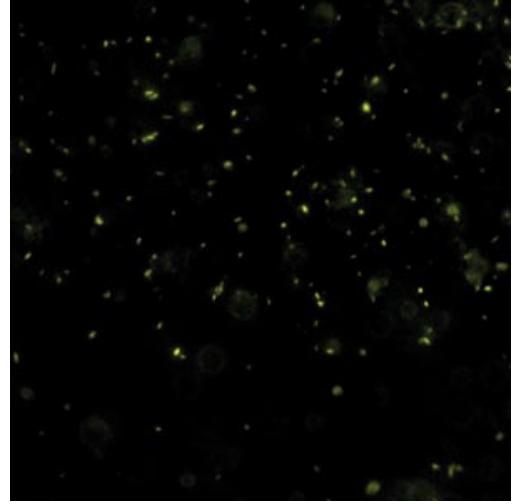

Figure 6. Epi-fluorescent image of bacteria spread on the funnel arry..

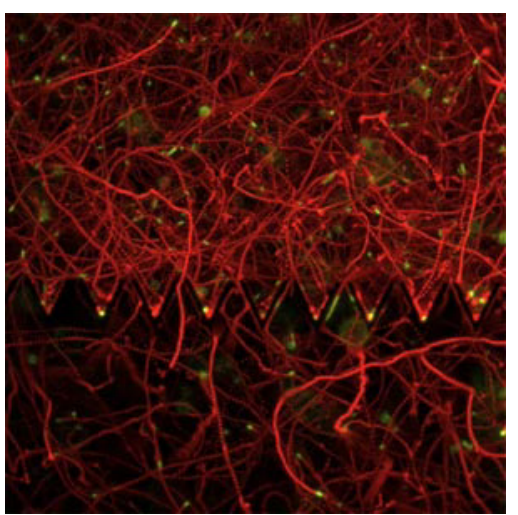

Figure 7. Tracks of bacteria observed after swimming in the funnel array.

bacteria, in this case the bacteria E. coli using expression of green fluorescent protein (GFP) to easily track the bacteria. This is more like a thermally driven system, since in the absence of a chemotactic gradient motile bacteria at low concentrations are believed to basically perform a random walk, ${ }^{2}$ which is not the same as brownian motion but one would hope close enough to test the pumping ability of a passive funnel. Fig. ?? presents pictures of the microfabricated funnel array and the resultant bacterial movement as they randomly swam in the array. It is clear that, as in the shaken box, the swimming bacteria have concentrated themselves. This might seem like a substantially greater challenge to the Second Law of Thermodynamics!

But it isn't a challenge to the Second Law, of course. Although the bacteria may swim in random directions, they do indeed swim and therefore do work, unlike a brownian particle which does no work. Since the bacteria do work, they can decrease the entropy of the system, just like a swimming rubber ducky, one of the simplest toys of them all, can indeed also concentrate themselves in a funnel array, as you can see if you actually put some rubber duckies in a wash basin with some simple funnels. ${ }^{3}$ The challenge actually is to a deeper issue in physics, and it connects to living systems, namely the reversibility of time. The bacteria move in a much different world in terms of forces and dissipation rates then do the duckies in the pan. ${ }^{4}$ As we have discussed earlier, ${ }^{3}$ technically we are dealing in general with the non-linear Navier-Stokes equation when we ask how cells (or rubber duckies) propel themselves through a viscous liquid:

$$
m\left[\frac{\partial \vec{v}}{\partial t}+\vec{v}(\nabla \bullet \vec{v})\right]=-\zeta \vec{v}+\nabla P
$$

where $m$ is the mass of the object, $\vec{v}$ is the velocity of the object, $\zeta$ is the coefficient of friction and $P$ is the local 


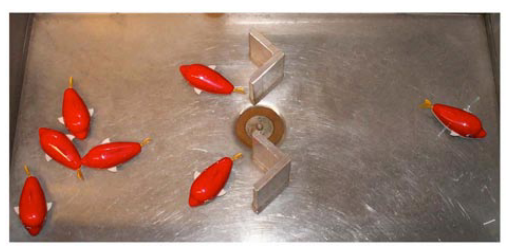

Figure 8. Rubber duckies selfconcentrate themselves at high Reynolds number in a funnel array.

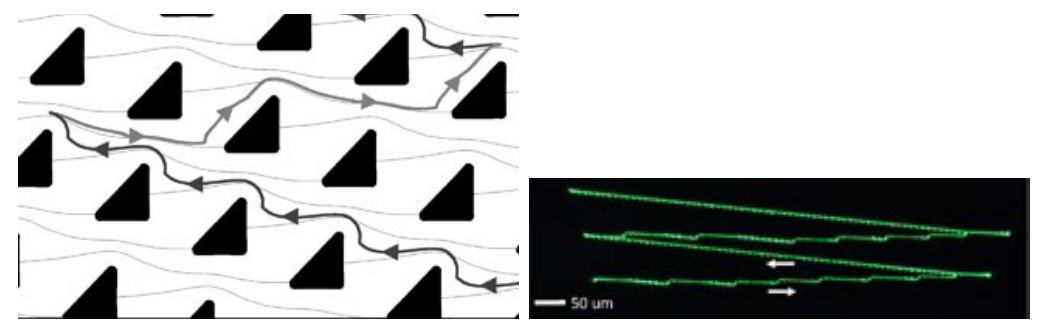

Figure 9. Using double-broken sym- Figure 10. Realization of the ratchmetry to obtain true ratcheting mo- eting motion in symmetrical flow. tion at low Re: basic scheme.

pressure. The Reynolds number, one of many dimensionless quantities of use in hydrodynamics, is a measure of the rate at which momentum is carried by the object to the rate it is lost to the liquid. At low Reynold's numbers however the situation is different, since the inertial $\frac{\partial \vec{v}}{\partial t}$ term is small compared to the viscous damping term and the spatial derivative of $\vec{v}(\nabla \bullet \vec{v})$ turns the Navier-Stokes equation to:

$$
m \nabla^{2} \vec{v}=-\zeta \vec{v}
$$

The Navier-Stokes equation at low Re has the same dynamic behavior if time is reversed and if the forces are reversed in sign: that is the displacement of particles moves back to where they were originally at time $t=0$ if after a time $t_{\text {reverse }}$ the forces are reversed in sign, which is the same as running time backwards. The problem then becomes this: if the bacteria are running in a low Re world, then why do the funnels operate in a time-irreversible manner? The answer is simple: both the Navier-Stokes Eq. 2 and the low Re version Eq. 2 are incomplete: they are only concerned with fluid flow in an unbounded medium. When are add boundaries and structures, as we do in our microfabrication technology, when an object nears a wall (how close "near" is remains a difficult subject) then additional non-hydrodynamic forces come into play that are not included in Eq. , and these forces break time-reversal symmetry. Time now moves forward in one direction. This breaking of symmetry via use of microfabrication of surfaces and the forces objects exert when they near asymmetrical surfaces can be of great use if you manage to have clever students.

For example, a recent student in our lab, Kevin Loutherback, had the idea to double-break symmetry in a device invented in our lab called (eventually) the Bump Array. ${ }^{5}$ The Bump Array was an example of breaking symmetry to get time-irreversible movement at low Re by simply shifting a rows of posts sequentially by a fractional distance $\epsilon$ from row to row. This made the Bump Array capable of separating objects of differing sizes with remarkable precision. But there are further degrees of symmetry breaking that can be done. In Kevin's case, the posts where turned into right-angled triangles from circular posts. This kind of symmetry breaking meant that now the bump array could not only separate posts, but it could also ratchet particles up and down even in an oscillating symmetric flow pattern. ${ }^{6}$ Using this kind of double-broken symmetry we can hope that truly innovative ways to sort materials can be developed. ${ }^{7}$

\section{MICROHABITATS AND EVOLUTION}

Although one could go on for a long time exploiting symmetries in making devices to understand how cells swim and figuring out clever ways to sort and fractionate cells, ultimately intellectually you want to go for bigger things. So far, I have described how toys acted as catalysts fro ideas. Now I will switch gears and and discuss how a Grimms Brother fairy tale help us exploit nano and microfabrication to address issues of evolution, and how that work seems to have resulted in the creation of a toy, rather than a toy leading to a new idea.

Let's start with the fairy tale, Goldilocks and the Three bears. ${ }^{8}$ Goldilocks sampled the three bear's wares for the "just right" combination of taste, fit and comfort. Like Goldilocks' need for the just right parameters, evolution proceeds most rapidly when there is the just right combination of a large number of mutants and rapid fixation of the mutants. ${ }^{9}$ We show here using a two-dimensional micro-ecology that it is possible to fix resistance to the powerful antibiotic ciprofloxacin (Cipro) in wild-type E. coli in 10 hours through a combination of extremely high population gradients, which generate rapid fixation, convolved with the "just right" level of 

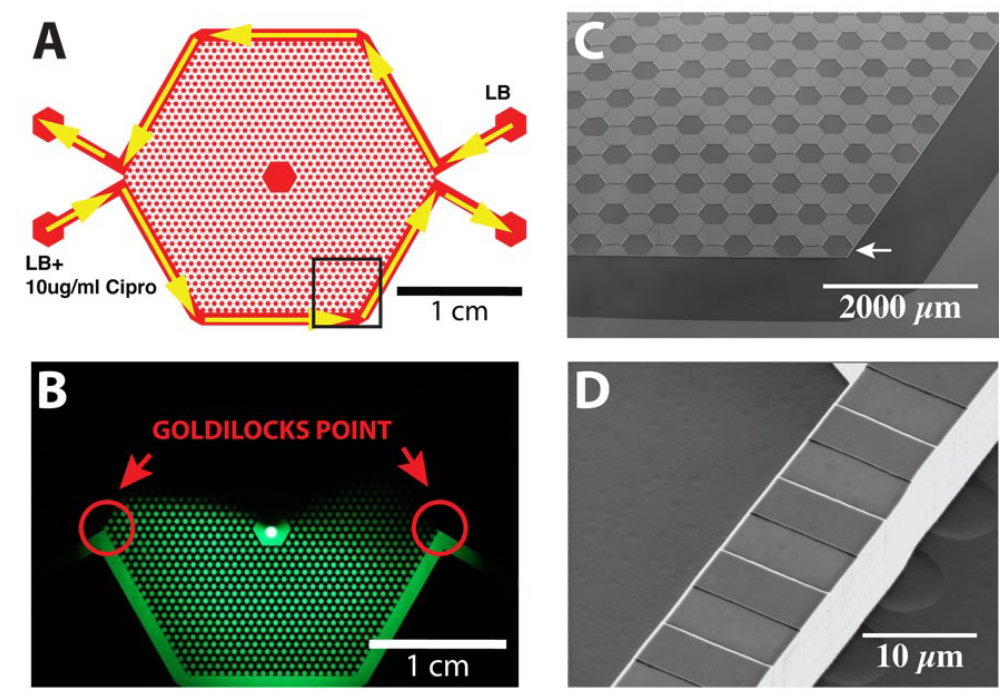

Figure 11. (A) An overview of the entire micro-ecology, showing the flow of the nutrient streams and the nutrient+Cipro containing streams. The nutrient stream is $\mathrm{x} 1 \mathrm{LB}$ broth, while the nutrient+Cipro stream is $\mathrm{x} 1 \mathrm{LB} \mathrm{broth}+10 \mu \mathrm{g} / \mathrm{mL}$ Cipro. (B) Image of the expected Cipro concentration using the dye fluorescein as a marker. The asymmetry of the pattern at the Goldilocks points is due to the direction of the flow. (C) Scanning electron microscope (SEM) image of the area of the array in (A) outlined by the box. Each hexagon is etched down 10 microns, the interconnecting channels are 10 microns deep and 10 microns wide. (D) SEM image of the nanoslits at the micro-ecology periphery. The nanoslits are etched down $100 \mathrm{~nm}$ and are 6 microns wide and 10 microns long.

antibiotic which generates a large number of mutants and the motility of the organism. Although evolution occurs in well-stirred chemostats without such Goldilocks conditions, ${ }^{10}$ natural environments are rarely well stirred in nature, as Darwin realized on the Galapagos Islands. ${ }^{11}$ For complex environments such as the Galapagos Islands, spatial population gradients and movement of mutants along these population gradients can be as important as genomic heterogeneity in the setting the speed of evolution. The design of our micro-ecology is unique in that it provides two overlapping gradients, one an emergent and self generated bacterial population gradient due to food restriction and the other a mutagenic antibiotic gradient. Further, it exploits the motility of the bacteria moving across these gradients, which we have discussed above, to drive the rate of resistance to Cipro to extraordinarily high rates.

Micro-nanofabricated structures have been successfully used to study single cells by manipulating their microenvironment, ${ }^{12-14}$ but it is still challenging to realize a controllable complex fitness landscape as we seek here. Our previous experiments have been relatively simple 1-D stress experiments. ${ }^{15,16}$ In this section, work done primarily by my student Qiucen Zhang, the Galapagos Islands of Darwin have been shrunk down to a $2 \mathrm{~cm}$ diameter interconnected network of 1200 hexagonal microhabitats, each of size 200 microns diameter, which can contain up to several thousand bacteria as shown in the Fig. 11A. Nutrients circulate around half the perimeter of the device, while nutrients + Cirpo circulate around the other half of the device, generating gradients of the antibiotic within the array of microhabitats (Fig. 11A.) The nanoslits (Fig. 11D) restrict food flow and also can generate emergent population gradients even in the absence of the antibiotic. 10 micron wide channels connecting the microhabitats allow for motion of bacteria between different metapopulations, a critical aspect of movement along the gradient once stress resistance has evolved in a local metapopulation.

Figure 12 shows the progression of resistance of wild-Type E. coli at a low-flow flow rate in each channel of 3 $\mu \mathrm{l} /$ hour over 20 hours. When bacteria are inoculated into the center of the device, chemotaxis due to consumption of nutrients at low flow rates quickly drives them to the perimeter of the device to form tight population gradients against the nano-slits. At the Goldilocks point (red circle, Fig. 12(A) there is a combination of high population gradient and high mutation rates. In this experiment the concentration of Cipro flowing along the bottom side of the device is extremely high, $10 \mu \mathrm{g} / \mathrm{ml}$ approximately 200 times MIC (MIC is the minimum inhibitory 

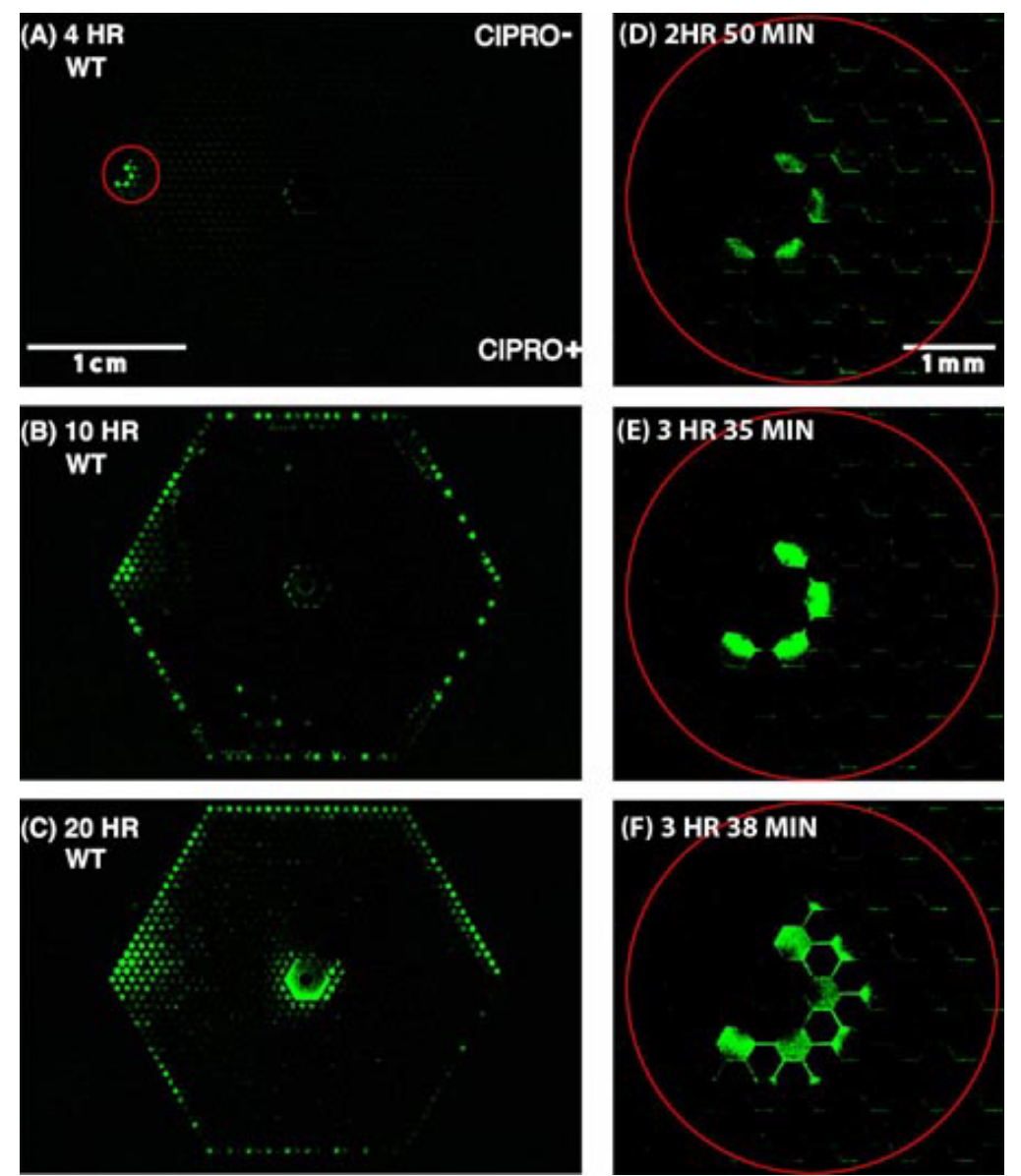

Figure 12. (A) Ignition of resistance to Cirpro at the Goldilocks point 4 hours after inoculation. (B) Spread of resistant bacteria around the periphery of the micro-ecology at 10 hours after inoculation. (C) Return of Cipro resistant bacteria to the center after 20 hours. The dynamics of the resistant bacteria at the Goldilocks point is shown with higher time resolution in (D)-(F) during the ignition of resistance over a 1 hour period.

concentration of Cipro. Yet, as Fig. 12 (A) shows there is ignition of resistance at the Goldilocks point and subsequent rapid movement of resistant bacteria around the periphery of the device Fig. 12(B) and invasion back to the center in 20 hours (Fig. 12(C). The basic reason for this invasion of resistance is the huge fitness advantage for mutant resistant $E$. coli in a micro-environment where the food reservoir is nearby and no other sensitive competitors can live. Since the device is composed of small micro-habitat patches, the resistant $E$. coli can rapidly fix in these small populations. Fig. 12(D)-(F) shows that the origin of resistance at 3 hours after inoculation is highly localized at the Goldilocks point initially and spreads rapidly outwards driven by cell motility. The position of the gradients relative to the apex hexagons sensitively determines where the Goldilocks points are.

Darwin transformed biology, but he provided no analytical template and we still have experimentally a great deal to learn about the dynamics of evolution, particularly under the response to stress. ${ }^{17}$ Fortunately we can analyze the resistance dynamics here and provide an analytical framework. There are several parameters that are relevant to enhancement of the evolution of resistance in mutagenic stress gradients: (1) the $N_{h}$ : population size in a high stress microhabitat, (2) the stress induced mutation rate $\mu^{*}$, (3) the net velocity of bacteria $\vec{v}$ along gradient and (4) the time before entering stationary phase $\tau,(5)$ the doubling time $t_{1 / 2}$ of the mutant bacteria in the presence of the antibiotic, (6) the fitness advantage of the resistant bacteria, $s$. The time $T_{e}$ for 


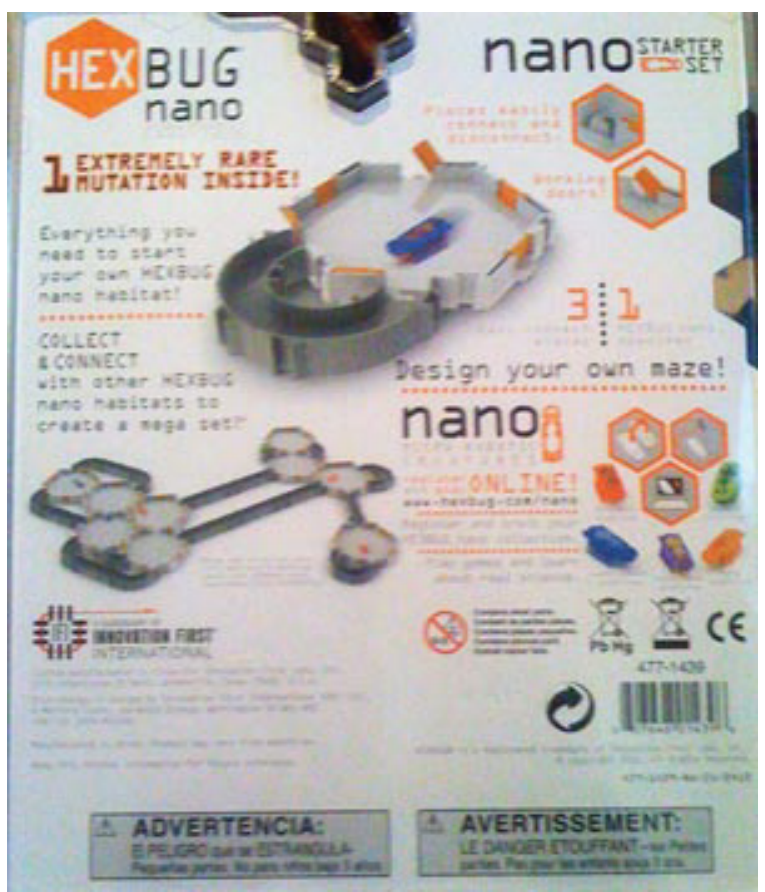

Figure 13. The HexBug Nanohabitat toy box.

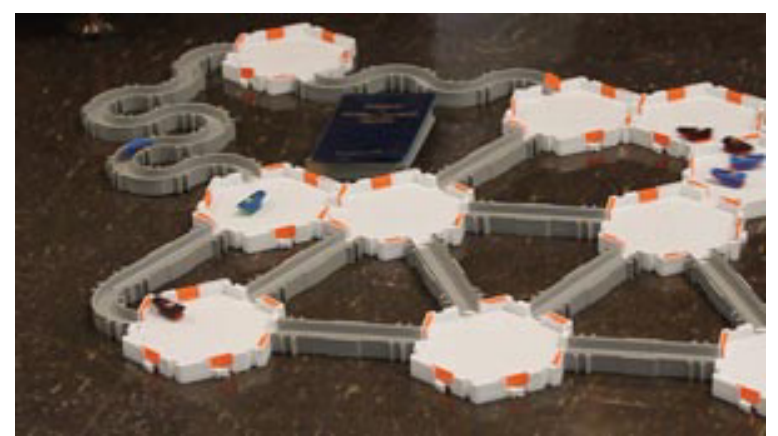

Figure 14. An assembled hex bug array.

the adaptive mutants to reach a frequency that assures escape from stochastic loss ${ }^{18}$ can be estimated to be:

$$
\frac{1}{T_{e}}=N_{h} \mu^{*}+\left(-\nabla N_{h} \cdot \vec{v}\right) \tau \mu^{*}
$$

The time to fix $T_{f}$ for a mutant is expected to scale as: ${ }^{19}$

$$
T_{f} \sim \frac{\ln \left(N_{h} \times s\right)}{s} \times t_{1 / 2}
$$

The total time $T_{\text {total }}$ then expected for the emergence of a fixed population of resistant bacteria is the $T_{e}+T_{f}$. We know that $N_{h}$ is approximately $10^{2}$ from the images in Fig. 12 , and that $\nabla N_{h} \sim 10 /$ micron, $\tau$ is about $10^{4}$ s from the 96 well plate time course measurements, $\vec{v}$ is about $10 \mu \mathrm{m} / \mathrm{s}, \mu^{*}$ (stress induced mutation rate) is about $10^{-5}$ mutants/viable cells/day. ${ }^{20}$ The fitness advantage $s$ can be roughly estimated as $M I C_{\text {resistance }} / M I C_{\text {wild-type }} \sim$ 20. Together, this analysis predicts that $T_{\text {total }} \sim 3$ hours, greatly reduced to the presence of the population gradients and Cipro-induced mutations, and is roughly the time observed in our experiments.

About the time we were doing these experiments, I was passing a toy on Martha's Vineyard and something caught my eye: a package with a lot of the words I have used in the above section: hexagonal, maze, mutation, bug (I assume here a bacterium): this looked strangely familiar! In fact, the basic design of my hexagonal evolution maze was made while I was on Sabbatical at the Hong Kong University of Science and Technology, where I gave a few talks on my ideas. This all looked very much like somebody had made a toy out of my basic evolution design. Of course I had to buy the toy (they are now freely available at many places in the US) and assemble my very own macro version of my original micro version of an evolution reactor. Toys rule.

\section{Acknowledgments}

This work was performed in part at the Cornell NanoScale Facility, a member of the National Nanotechnology Infrastructure Network, which is supported by the National Science Foundation (Grant ECS-0335765). This material is based upon work supported by the National Science Foundation under Grant No. 0750323. This work was also supported by the Department of Defense NDSEG fellowship program and the Natural Sciences and Engineering Research Council of Canada. The research described was supported by Award Number U54CA143803 
from the National Cancer Institute. The content is solely the responsibility of the authors and does not necessarily represent the official views of the National Cancer Institute or the National Institutes of Health.

\section{REFERENCES}

[1] Austin, R. H., Xie, A. H., Fu, D., Warren, W., Redlich, B., and van der Meer, L., "Tilting after dutch windmills: probably no long-lived davydov solitons in proteins," Journal of Biological Physics 35(1), 91101 (2009). 424EK Times Cited:1 Cited References Count:22.

[2] Codling, E. A., Plank, M. J., and Benhamou, S., "Random walk models in biology," Journal of the Royal Society Interface 5(25), 813-834 (2008). 317GW Times Cited:40 Cited References Count:102.

[3] Galajda, P., Keymer, J., Dalland, J., Park, S., Kou, S., and Austin, R., "Funnel ratchets in biology at low reynolds number: choanotaxis," Journal of Modern Optics 55(19-20), 3413-3422 (2008). 385GS Times Cited:3 Cited References Count:15.

[4] Purcell, E. M., "Life at low reynolds-number," American Journal of Physics 45(1), 3-11 (1977). Cs643 Times Cited:692 Cited References Count:10.

[5] Huang, L. R., Cox, E. C., Austin, R. H., and Sturm, J. C., "Continuous particle separation through deterministic lateral displacement," Science 304(5673), 987-990 (2004). 820IV Times Cited:261 Cited References Count:24.

[6] Loutherback, K., Puchalla, J., Austin, R. H., and Sturm, J. C., "Deterministic microfluidic ratchet," Physical Review Letters 102(4), - (2009). 401YC Times Cited:5 Cited References Count:18.

[7] Morton, K. J., Loutherback, K., Inglis, D. W., Tsui, O. K., Sturm, J. C., Chou, S. Y., and Austin, R. H., "Crossing microfluidic streamlines to lyse, label and wash cells," Lab on a Chip 8(9), 1448-1453 (2008). 354YO Times Cited:16 Cited References Count:22.

[8] Opie, I. and Opie, P., "The classic fairy tales," Oxford University Press, 199200 (1974).

[9] Pulliam, H. R. and Danielson, B. J., "Sources, sinks, and habitat selection - a landscape perspective on population-dynamics," American Naturalist 137, S50-S66 (1991).

[10] Barrick, J. E., Yu, D. S., Yoon, S. H., Jeong, H., Oh, T. K., Schneider, D., Lenski, R. E., and Kim, J. F., "Genome evolution and adaptation in a long-term experiment with escherichia coli," Nature 461(7268), 1243-U1274 (2009).

[11] Keynes, R. D., "Charles darwin's beagle diary," Cambridge University Press (1988).

[12] Weibel, D. B., DiLuzio, W. R., and Whitesides, G. M., "Microfabrication meets microbiology," Nat Rev Micro 5, 209-218 (Mar. 2007).

[13] Whitesides, G. M., "The origins and the future of microfluidics," Nature 442, 368-373 (July 2006).

[14] Beebe, D. J., Mensing, G. A., and Walker, G. M., "PHYSICS AND APPLICATIONS OF MICROFLUIDICS IN BIOLOGY," Annual Review of Biomedical Engineering 4(1), 261-286 (2002).

[15] Keymer, J. E., Galajda, P., Muldoon, C., Park, S., and Austin, R. H., "Bacterial metapopulations in nanofabricated landscapes," Proceedings of the National Academy of Sciences 103, 17290 -17295 (Nov. 2006).

[16] Keymer, J. E., Galajda, P., Lambert, G., Liao, D., and Austin, R. H., "Computation of mutual fitness by competing bacteria," Proceedings of the National Academy of Sciences 105, 20269 -20273 (Dec. 2008).

[17] Michel, B., "After 30 years of study, the bacterial sos response still surprises us," Plos Biology 3(7), 1174$1176(2005)$.

[18] J.H., G., "The causes of molecular evolution," Oxford University Press (1991).

[19] Karasov, T., Messer, P. W., and Petrov, D. A., "Evidence that adaptation in drosophila is not limited by mutation at single sites," Plos Genetics 6(6), - (2010).

[20] Cirz, R. T., Chin, J. K., Andes, D. R., de Crcy-Lagard, V., Craig, W. A., and Romesberg, F. E., "Inhibition of mutation and combating the evolution of antibiotic resistance," PLoS Biol 3, e176 (May 2005). 